\title{
Precision Assembling and Hybrid Bonding for Micro Fluidic Systems
}

\author{
Agathe Koller-Hodac, Manuel Altmeyer, and Silvio Walpen \\ Institute for Laboratory Technology, University of Applied Sciences Rapperswil, \\ Oberseestrasse 10, \\ CH-8640 Rapperswil, Switzerland \\ \{agathe.koller, manuel.altmeyer, silvio.walpen\}@hsr.ch
}

\begin{abstract}
An automated process is described to assemble highly functionalized micro fluidic cartridges. Different materials such as glass, silicon and thermoplastics can be bonded using adhesive gaskets. The assembling and bonding sequence is realized at room temperature so that the biological function of the cartridge is fully preserved. Several control tests for alignment and orientation are integrated into the process so that a precise and reliable assembly using robotic equipment can be achieved.
\end{abstract}

Keywords: Micro-assembly, hybrid bonding, micro fluidics, micro-gripper.

\section{Introduction}

Miniaturization of biological assays is a major trend in the pharmaceutical and life science industry. This allows not only the reduction of expensive reagents, but also enables the integration of additional functions such as mixing or dosing into micro fluidic cartridges. Unfortunately the integration of such advanced functionalities fails in production due to the lack of suited reliable automated assembly technologies that are also able to maintain the biological function of the micro fluidic device.

Due to increasing demand for optical and micro-electro-mechanical Systems, micro-assembly platforms and tools have been developed in recent years [1]. Considering the bonding process in an industrial environment, interesting approaches have been reported in the semiconductor sector. The automated bonding is mainly based on a thermal process.

In life sciences, especially in the micro fluidic domain, conventional automated processes are not applicable to the bonding of micro fluidic devices when biomolecules must be deposited onto the chip prior to sealing. Also new assembly issues must be addressed that are often not compatible with existing processes. Until now, assembly of complex micro fluidic components made of different materials is done either manually or semi-automatically by using a range of heterogeneous tools such as microscopes, precision stages or fixtures. However, this approach suffers from a lack of flexibility, scalability and reproducible quality. The absence of appropriate equipment prevents a mass production and a cost-effective commercialization of micro fluidic components. Therefore, there is an increasing need for innovative and low-cost processes for automated high precision assembly of hybrid micro fluidic components, such as plastic and glass. 
Our main research and development focus lies on a fabrication process which maintains the biological function throughout the entire automated assembly of the micro fluidic product. The highly functional cartridges require the integration of additional specialized elements into the plastic micro fluidic device, such as chemically functionalized detection units made of silicon or glass. Reliable hybrid bonding is becoming the key issue in cartridge production. Biocompatibility, low temperatures and no out-gassing of any material in the cartridge are just few points that have to be fulfilled. In addition the assembly and bonding process must be cost efficient and adapted to different cartridge geometries.

This paper presents an automated assembly process for multi-layer micro fluidic cartridges consisting of a scaffold, a double-sided adhesive gasket and a pre-coated chip. Special emphasis has been on fully preserving function of pre-coatings during the bonding process. No heat or ultraviolet light is necessary during assembly. For a systematic evaluation of the process, alignment monitoring as well as quality control of the final device have been developed.

\section{Assembly Process and Layout}

In the first section, the different layers of micro fluidic cartridge are introduced. In the second section, the sequential steps of the assembly process are highlighted. Special emphasis lies on the correct orientation and precise alignment of components before assembling. Finally, the quality control of the final cartridge is described.

\subsection{Cartridge Components}

Components of the micro fluidic cartridge have been designed to facilitate the assembly process. Several alignment features are implemented on the scaffold and the adhesive gasket and its protective foil have been specially developed to support the automated assembly. Type and thickness of the gasket material turned out to be essential parameters to achieve high bonding quality. The glass chip has a quadratic shape with no reference marks and is pre-coated with biomolecules.



Fig. 1. Components of micro fluidic cartridge

Scaffolds and chips are supplied on trays which are placed within the robotic cell. Supply of adhesive gaskets requires special equipment and methods, since doublesided tape is very delicate to handle. The developed solution is based on an automated feeding system with foil rolls. 


\subsection{Precision Assembling}

A major challenge associated to this development is to perform reliable and accurate assembling of micro fluidic parts without requiring an expensive apparatus. The production cell, as shown in Fig. 2, includes several mounting modules as well as storage trays for scaffolds and chips. The size and complexity of the assembling and hybrid bonding processes made it necessary to define sub processes and integrate quality control checks.
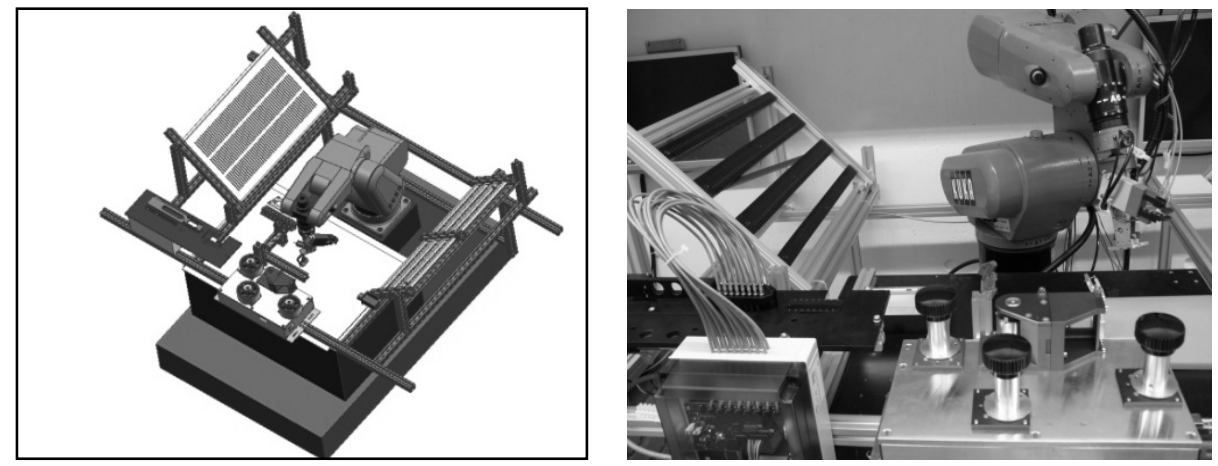

Fig. 2. The robotic cell includes an articulated robot (middle), two supply systems for chips and scaffolds (left and right of the robot), a feeding system for adhesive gaskets (front) and a sealing test module (front left)

The experimental setup includes a standard articulated industrial robot with six degrees of freedom for coarse positioning tasks. The robot kinematics with six rotational axes allow placing chips and especially scaffolds in a number of positions and orientations required to perform assembling and testing operations. Moreover, the articulated robot Kuka KR3 has an adequate workspace to supply and storage sufficient parts for long production autonomy. Fine positioning is achieved using a hybrid gripper, which design allows normal pick and place operations as well as accurate joining of several micro fluidic part layers. A vacuum system allows grasping chips at the outside edge, while entirely preserving surface coating with bio-molecules.

The Fig. 3 describes the complete assembling process which has been split into sub processes. All cartridge components are supplied and automatically mounted in the robotic cell. Exact position or orientation of each component is controlled before each assembling step.

The robot picks the parts out of the trays and performs initial handling tasks. Since transparent chips have an asymmetric coating, their orientation has to be detected for correct assembling. A standalone vision system identifies the chip orientation and transmits data to the robot control. A dedicated module for automatically supplying and preparing adhesive gaskets has been developed and integrated into the robotic cell.

The assembling quality of the micro fluidic cartridge plays an important role in the application assay. In particular, a part alignment accuracy better that $20 \mu \mathrm{m}$ is 


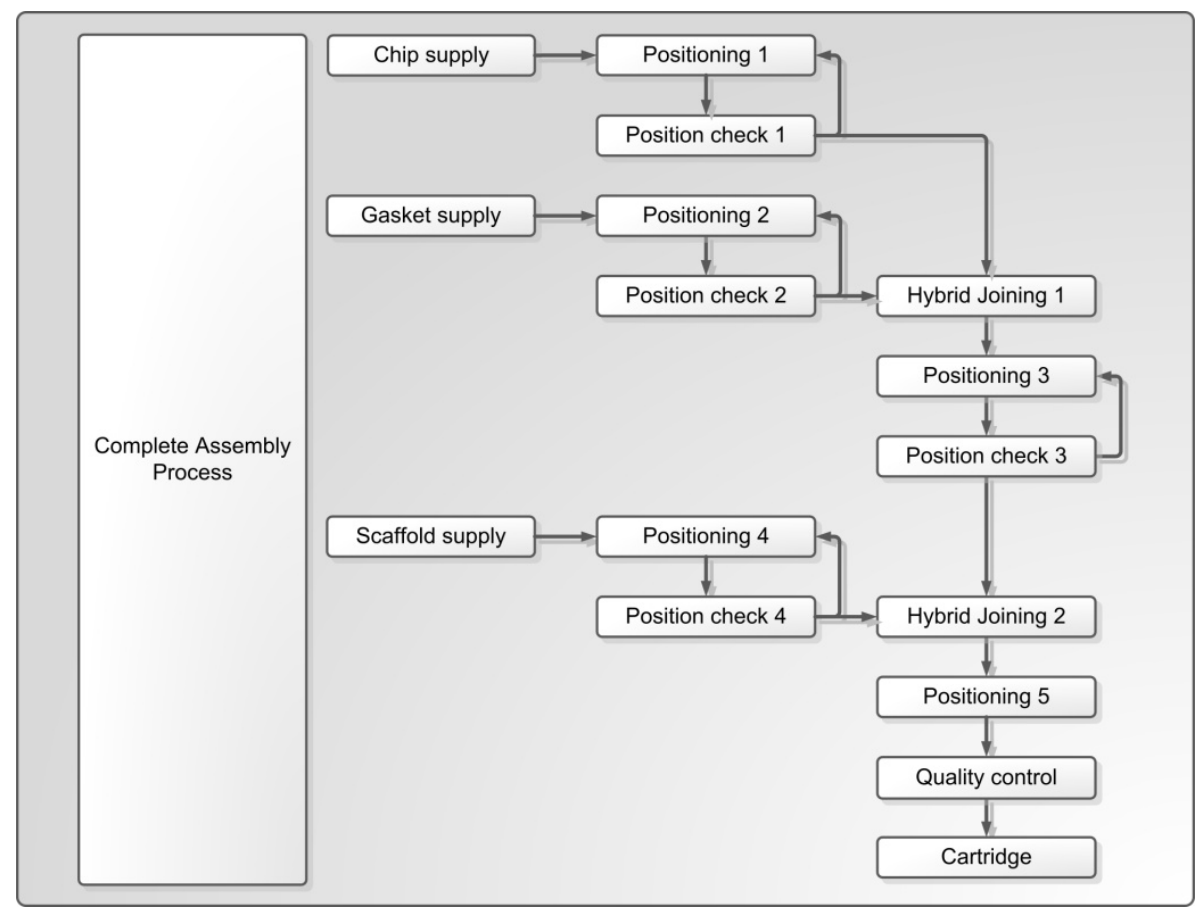

Fig. 3. Description of the assembly process: Exact component position is controlled before each hybrid bonding step

required, which is higher than the accuracy of $\pm 0.05 \mathrm{~mm}$ provided by the robot [2]. Therefore, the first joining step requires position monitoring based on a control loop consisting of vision sensor, image processing software and robot control. This control algorithm is based on the look-and-move principle [3], [5]. After this first joining step, chip and gasket are precisely positioned on the hybrid gripper.

The second joining step combining chip and gasket with the scaffold is solved using the mechanical mounting support of the hybrid gripper. Its guiding balls interact with the scaffold grooves, leading chip and gasket to their correct position on the scaffold. In the last process step, the assembling quality of the micro fluidic cartridge is assessed using a sealing check module. The critical steps are described in details in the following sections.

\subsubsection{Chip Orientation Detection}

The glass chip consists of a quadratic plate without additional mechanical or optical orientation marks. This must be correctly orientated due to pre-coating asymmetry. Placed in the inspection cradle as shown on Fig. 4, the chip has eight possible orientations: Four ninety degree turning steps and front- or backside. The chip being the most valuable part of the assembly, a full orientation control is required to ensure a correctly assembled cartridge at a minimal yield. 


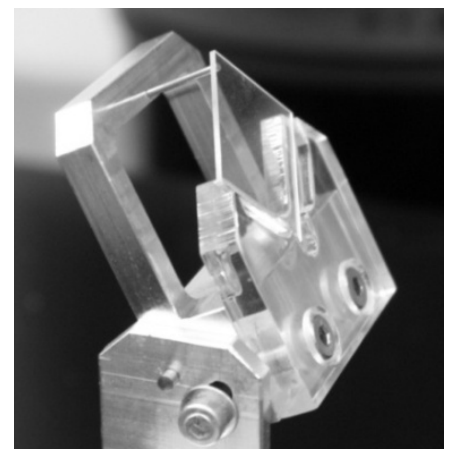

Fig. 4. Chip Inspection Cradle

The coating on the crystal clear glass substrate is highly translucent. The coating pattern reflects green whereas the background appears violet. A reliable and stable detection has been obtained by using a color camera system.

Based on the preprocessed camera information, the image processing algorithms can detect the ninety degree rotation and can differentiate between front and back sides. Following the concept of decentralized modules, a smart camera which contains both camera and image processing is used here.

\subsubsection{Gasket Supply}

A major step in the assembly process is the supply and handling of double-sided adhesive gaskets. Besides the tape material and thickness, the supply procedure is essential to obtain reliable bonding results. Based on preliminary tests, the gasket supply on an endless carrier foil covered by an additional protection foil has proven to be the best solution. Perforations like on $35 \mathrm{~mm}$ cinema films or analog picture films have been laser-cut at the side of carrier foils to provide reference marks.

The tape feeding system is shown on Fig. 5. The bonding area for gasket and chip is located on a glass plate (A). The roller of small diameter (B) has notched dents matching the perforations of the carrier foil. Being driven by a controlled stepper

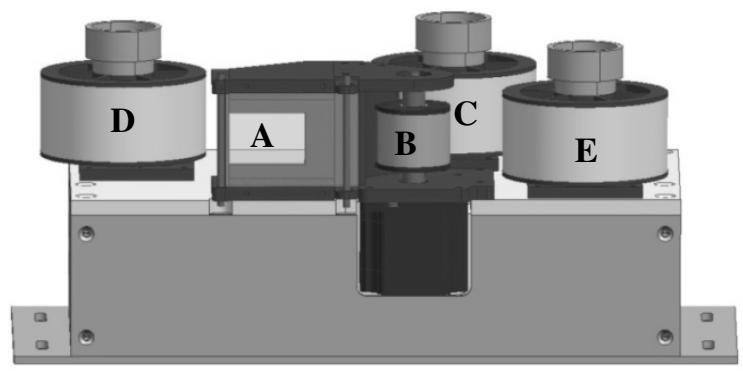

Fig. 5. The tape feeding system supplies adhesive gaskets automatically. Gaskets are provided on foil rolls and are covered by protection foils. Before hybrid bonding, one gasket is moved to the joining area while the protection foil is removed. 
motor, it is responsible for the gasket basis positioning. The spender roller (C) in the center unwinds the adhesive gasket. The bobbin (D) reels the blank carrier foil after chip and gasket bonding is performed in the joining area. The bobbin (E) reels the protection foil. All these three rollers are motor driven to ensure the correct foil tension at the bonding position and to avoid uncontrolled unwinding.

\subsubsection{Chip Alignment}

The chip has been grasped by the robot vacuum gripper and the adhesive gasket has been provided by the tape feeding system. The next process step is the delicate assembling of the chip to the adhesive gasket. A vision system detects the exact position of the adhesive gasket through the glass plate of the tape feeder. Based on a calibrated picture, the position offset of the gasket is determined and provided to the cartesian controller of the robot. The control algorithm illustrated in Fig. 6, based on the lookand-move principle, checks and eventually corrects the position of gripper relative to the adhesive gasket, before the chip is bonded to the gasket.

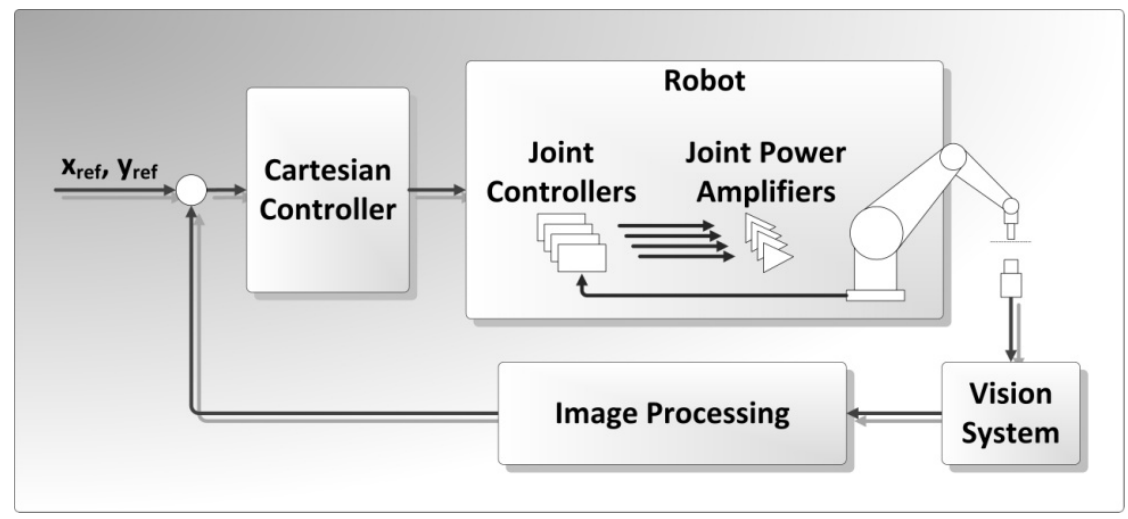

Fig. 6. Look-and-move control loop: a vision system provides exact information about gasket position. The robot aligns the chip to the adhesive gasket before hybrid bonding.

\subsubsection{Scaffold Alignment}

The precise positioning of the scaffold with respect to the chip is solved mechanically using guiding balls glued onto the hybrid gripper. These three balls are aligned to the grooves designed in the injection molded scaffold [4]. The combination of grooves and balls hinders the degrees of freedom of the scaffold relative to the gripper, leading to a precise alignment of both components.

The joining sequence is illustrated in Fig. 7. The gripper with its guiding balls moves close to the scaffold which lies on an air cushion area. The scaffold is floating when the air cushion is activated. The groove arrangement results in an exact alignment between chip and scaffold. In a next step, the gripper element which holds chip and gasket moves linearly downward the scaffold and joins both components so that the micro fluidic cartridge is finally assembled. 

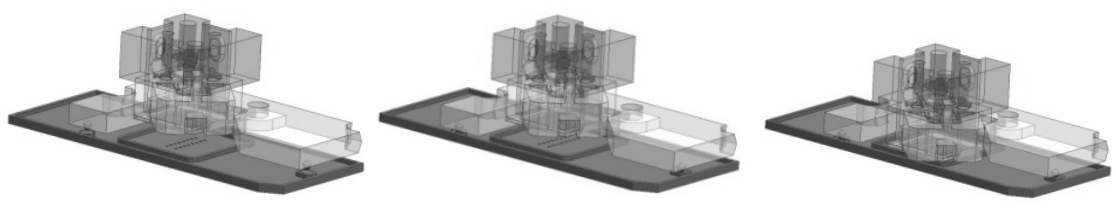

Fig. 7. Assembling sequence for chip and scaffold: (1) the robot gripper gets close to the scaffold, (2) the scaffold is floating on an air cushion (3) the gripper is actuated linearly in order to bond chip to scaffold

\subsection{Quality Control}

Micro fluidic cartridges have to be mounted with high precision due to small dimensions of micro fluidic channels and interfaces. As already described, several modules have been integrated into the robotic cell to control and adjust position, alignment and orientation during the assembly process. An additional module performs automatically a sealing test of the final cartridge. This test consists in checking pressure in all micro fluidic channels. Assembled cartridges which fail the sealing test have been discarded.

\section{Conclusion}

An innovative yet reliable method has been achieved for automatically assembling micro fluidic parts composed of several layers.

A robotic cell has been developed and realized to assemble highly functionalized micro fluidic cartridges in an industrial environment. Experimental results have shown that hybrid bonding of different materials - such as micro-structured plastic parts and high-quality, bio-functionalized silicon or glass elements - can be performed using adhesive gaskets. The whole assembling and bonding process is realized at room temperature so that the biological function of the cartridge is preserved.

While the whole automated process is based on a standard industrial robot, the precise assembly of multiple layers has been made possible through an innovative gripper design. Further, a vision based look-and-move control approach allows the robot to adjust position relative to micro fluidic layers, in order to precisely align micro fluidic channels to pre-coated spots.

\section{Acknowledgement}

Our sincere thank to Markus Lüthy, Edi Krüttli und Kurt Eggmann from Weidmann Plastics Technology AG for their support and part manufacturing.

Grateful thanks also to Max Wiki from Dynetix AG and to Stephane Follonier, Janko Auerswald und Qun Lai from CSEM SA for their expertise and important contribution to the project.

This project has been supported by the Swiss Innovation Promotion Agency (CTI). 


\section{References}

1. Hollis, R.L., Gowdy, J., Rizzi, A.A.: Design and Development of a Tabletop Precision Assembly System. In: Mechatronics and Robotics (MechRob 2004), Aachen, Germany, September 13-15, pp. 1619-1623 (2004)

2. EN ISO 9283, Industrieroboter, Leistungskenngrössen und zugehörige Prüfmethoden. Beuth-Verlag, Berlin (1999)

3. Conticelli, F., Allotta, B.: Two-Level Visual Control of Dynamic Look-and-Move Systems. In: IEEE lnternational Conference on Robotics \& Automation, San Francisco, CA (2000)

4. Wiendahl, B., Lotter, H.P.: Montage in der industriellen Produktion - Ein Handbuch für die Praxis. Springer, Heidelberg (2006)

5. Schöttler, K., Raatz, A., Hesselbach, J., Wu, H.: Size-adapted Parallel and Hybrid Parallel Robots for Sensor Guided Micro-Assembly. In: Parallel Manipulators; Towards New Applications, pp. 225-244. I-Tech Publication and Publishing (2008) ISBN 978-3-902613-40-0

6. Schöttler, K., Raatz, A., Hesselbach, J.: Precision Assembly of Active Microsystems with a Size-Adapted Assembly System. In: Fourth International Precision Assembly Seminar (IPAS 2008), Chamonix, France, pp. 199-206. Springer, Boston (2008) 